\title{
Methicillin-resistant Staphylococcus aureus of the clonal lineage ST5-SCCmecll-t2460 was associated with high mortality in a Wuhan hospital
}

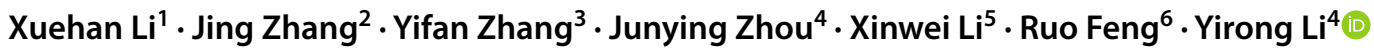

Received: 5 March 2021 / Accepted: 27 June 2021 / Published online: 8 July 2021

(c) The Author(s) 2021

\begin{abstract}
Methicillin-resistant Staphylococcus aureus (MRSA) is an important human pathogen that can cause serious infectious diseases. An emerging MRSA strain, ST5-SCCmecII spa-type-t2460 (SMRSA), has spread rapidly since its recent emergence in China, but little information is available about this lineage. In this study, 91 MRSA isolates were collected from patients treated in the Zhongnan Hospital, Wuhan University, from 2018 to 2019, and investigated for their molecular characteristics, antibiotic resistance profiles, and clinical characteristics. The predominant lineage, SMRSA, accounted for 37.4\% (34/91) of the isolates, followed by ST239-SCCmecIII-t030 (19.8\%, 18/91) and ST59-SCCmecIV-t437 (8.8\%, 8/91). In contrast to the latter two non-SMRSA (nSMRSA) lineages, which are among the main MRSA found in Chinese settings, SMRSA exhibited small colony variant (SCV) phenotype and had extremely high resistance rates to erythromycin (100.0\%), clindamycin (100.0\%), levofloxacin (100.0\%), tetracycline (97.1\%), moxifloxacin (97.1\%), and ciprofloxacin (100\%), but was more susceptible to rifampicin (resistance rate 3\%). The levels of white blood cells (WBC) and procalcitonin (PCT) and the 30-day mortality in patients infected with SMRSA were $(12.54 \pm 6.61) \times 10^{9} / \mathrm{L}, 0.66 \mathrm{ng} / \mathrm{mL}$, and $52.9 \%$, respectively, which were much higher than those in patients infected with nSMRSA. In addition, patients infected with SMRSA were more frequently admitted to the intensive care unit (ICU) and submitted to invasive procedures than those infected with nSMRSA. In conclusion, SMRSA showed SCV phenotype and exhibited multiple antibiotic-resistance profiles. In this study, SMRSA was associated with serious infections and poor prognosis. Compared with ST239, ST59, or other nSMRSA strains, patients infected with SMRSA strains have higher 30-day mortality, increased levels of inflammatory biomarkers, and more frequent ICU hospitalization and invasive procedures.
\end{abstract}

Keywords Methicillin-resistant Staphylococcus aureus · ST5-SCCmecII-t2460 • 30-day mortality · Procalcitonin · Antimicrobial susceptibility test

\section{Introduction}

Xuehan Li and Jing Zhang contributed equally to this work.

Responsible Editor: Agnes M.S. Figueiredo

Ruo Feng

fr@zzu.edu.cn

Yirong Li

liyirong838@163.com

1 Department of Laboratory Medicine, The Fifth Affiliated Hospital of Zhengzhou University, Zhengzhou, China

2 Department of Laboratory Medicine, Beijing Haidian Maternal and Child Health Hospital, Beijing, China
Methicillin-resistant Staphylococcus aureus (MRSA) is an important human pathogen that can cause a variety of diseases ranging from mild localized infections to severe

3 Department of Laboratory Medicine, The Affiliated Cancer Hospital of Zhengzhou University, Zhengzhou, China

4 Department of Laboratory Medicine, Zhongnan Hospital of Wuhan University, Wuhan, China

5 School of Basic Medical Sciences, Zhengzhou University, Zhengzhou, China

6 Department of Histology and Embryology, School of Basic Medical Sciences, Zhengzhou University, Zhengzhou, China 
systemic infections [1-3]. Since the first detection in 1961 in Europe, MRSA isolates have become a leading cause of nosocomial infection throughout the world [4]. The prevalence of MRSA across China has declined in recent years, but it remained at $33.6 \%$ in the first half of 2020 (www. chinets.com). Recently, diverse molecular techniques have been applied to monitor the emergence of pandemic clones [5], and the molecular and epidemiological characteristics of MRSA have been investigated exhaustively in China. The molecular characteristics of MRSA strains vary with the geographic regions and have been related to complications, disease severity, and mortality [6, 7]. Since 2013, SMRSA has emerged in China and has rapidly increased in number. In 2017, SMRSA even replaced ST239-SCCmecIII-t030 and ST5-SCCmecII-t002 becoming the predominant MRSA clonal lineage in Shanghai $[8,9]$. Meanwhile, our previous study showed that SMRSA was the predominant clone causing bloodstream infection in Wuhan [10].

The SMRSA strains were first found in South Korea causing an outbreak in 2007 [11]. In China, the prevalence of SMRSA has importantly increased in recent years. However, little information is available about the clinical characteristics and the evolutionary process of the SMRSA clone. Moreover, although many studies have investigated the molecular characteristics of MRSA in China [8, 12-14], few have explored the relationship between these molecular characteristics and the clinical prognosis of patients, especially for the predominant MRSA lineages. In this study, we identified some clinical features of SMRSA isolates and the clinical outcomes of SMRSA-infected patients in comparison with nSMRSA infections.

\section{Materials and methods}

\section{S. aureus isolates}

The study was performed at Zhongnan Hospital of Wuhan University. Ninety-one non-duplicate MRSA isolates were collected from the hospital's microbiology diagnostic lab. These isolates were derived from diverse clinical specimens of adult inpatients (age $>18$ years) who had cough, fever, and other clinical symptoms related to infections and were hospitalized between January 2018 and December 2019. The specimens included skin/soft tissue $(n=15,16.5 \%)$, sputum ( $n=38,41.8 \%)$, blood $(n=20,22.0 \%)$, and others (catheter tip, drainage liquid, ascites, and bronchoalveolar lavage fluid) $(n=18,19.8 \%)$.

Isolates were identified as $S$. aureus using conventional microbiological methods including Gram staining, catalase tests, and coagulase tests, and then further identified using the VITEK 2 Compact system and the VITEK 2 ASTGP67 Test Kit (bioMerieux, Inc., Durham, NC, U.S.A.).
Next, MRSA isolates were recognized by their resistance to cefoxitin and confirmed by the presence of the mecA gene [15]. All MRSA isolates were stored at $-80^{\circ} \mathrm{C}$ until further experiments. This study was approved by the Ethics Committee of Zhongnan Hospital of Wuhan University (No.2019126), which waived the requirement for informed consent because the details of patients with $S$. aureus infection were anonymized.

\section{Phenotypic and molecular characteristics of $S$. aureus}

All MRSA isolates were cultured on Columbia blood-agar at $37^{\circ} \mathrm{C}$. After $24 \mathrm{~h}$, the colony morphology was observed. Subsequently, a single colony was taken for chromosomal DNA extraction as described previously $[10,16]$. The extracted chromosomal DNAs were stored at $-20^{\circ} \mathrm{C}$ for staphylococcal protein A (spa) typing, multilocus sequence typing (MLST), and staphylococcus chromosomal cassette mec (SCCmec) typing according to previous studies [10, 17]. MRSA isolates that could not be classified as any known $\mathrm{SCC} m e c$ type were defined as nontypable.

\section{Antimicrobial susceptibility testing}

Antimicrobial susceptibility was determined by the VITEK 2 Compact system (bioMerieux, Inc., Durham, NC, U.S.A.). Fourteen antibiotics were tested, including cefoxitin, clindamycin, erythromycin, gentamicin, levofloxacin, linezolid, oxacillin, penicillin, rifampicin, trimethoprim/sulfamethoxazole, tetracycline, moxifloxacin, ciprofloxacin, and vancomycin. According to the results provided by VITEK 2, cefoxitin-resistant (cefoxitin minimum inhibitory concentration $\geq 8 \mu \mathrm{g} / \mathrm{mL}$ ) and $m e c A$-positive isolates were identified as MRSA. Isolates resistant to three or more antimicrobial classes were defined as multidrug-resistant $S$. aureus. ATCC 29,213 was used as the quality control organism, and the results were interpreted according to Clinical and Laboratory Standards Institute (CLSI) guidelines (CLSI M100-S29) [18].

\section{Clinical data collection}

We collected the following data retrospectively from the patients' hospital medical records: age, sex, underlying disease (the most common conditions were diabetes mellitus, malignancy, chronic renal disease, chronic pulmonary disease, neurologic, cardiovascular or hepatic disease), blood indicators, the levels of procalcitonin, outcome after 30 days of infection (survival or death), source of infection (skin/ soft tissue, blood, sputum, and other), and invasive procedures (trachea cannula, implantable venous access device, and catheter) performed within 1 month before infection. 
Based on medical records, the 91 MRSA isolates were categorized as community-acquired (CA)- or hospital-acquired (HA)-MRSA according to the previous definition [19]. CAMRSA refers to strains that were isolated within $48 \mathrm{~h}$ after hospital admission from an outpatient or an inpatient who had no medical history of MRSA infection, surgery, insertion of indwelling devices, or other risk factors for HAMRSA infection in the past year. HA-MRSA refers to strains obtained from patients who had been hospitalized for $48 \mathrm{~h}$ or longer.

\section{Statistical analysis}

Statistical analyses were performed using SPSS 22.0 (IBM, Armonk, NY, U.S.A.). Categorical variables were analyzed by univariate analysis using the chi-square or Fisher's exact tests. Continuous variables were analyzed by Student's $t$-test. All statistical tests were two-tailed.

\section{Results}

\section{Phenotypic and molecular characteristics of $S$. aureus}

As shown in Table 1, the MRSA strains were assigned into 15 sequence types, 23 spa types, and 5 SCCmec types. Of them, 4 isolates were classified as nontypable for SCCmec typing. Among the 91 MRSA isolates, SMRSA accounted for $37.4 \%$ (34/91), being the predominant clone, followed by ST239-SCCmecIII-t030 $(19.8 \%, 18 / 91)$ and ST59-SCCme$c \mathrm{IV}-\mathrm{t} 437(8.8 \%, 8 / 91)$, as determined by spa typing, MLST, and SCCmec typing.

After $24 \mathrm{~h}$, the 34 SMRSA strains all showed a similar phenotype characterized by small colony size, absence of pigmentation, and weak hemolytic activity on Columbia blood-agar. We speculated that the SMRSA strains were SCVs of S. aureus. We further isolated and subcultured some SCVs and confirmed that they were $S$. aureus using the VITEK 2 Compact system.

\section{Antimicrobial susceptibility}

The 91 MRSA isolates were tested for antimicrobial susceptibility. All strains were resistant to oxacillin, cefoxitin, and penicillin. No MRSA isolate was resistant to vancomycin or linezolid. The results of antimicrobial susceptibility tests for the other nine antibiotics are shown in Table 2. Most of the MRSA strains exhibited multiple antibioticresistance profiles. Specifically, $89.0 \%$ were multidrugresistant and more than $50 \%$ were resistant to seven of the
Table 1 Molecular characteristics of 91 MRSA isolates

\begin{tabular}{|c|c|c|}
\hline MLST (n) & SCCmec (n) & $\operatorname{spa}(\mathrm{n})$ \\
\hline \multirow[t]{3}{*}{ ST5(39) } & $\mathrm{II}(34)$ & $\mathrm{t} 2460(34)$ \\
\hline & II $(4)$ & $\mathrm{t} 311(4)$ \\
\hline & $\mathrm{II}(1)$ & t002(1) \\
\hline \multirow[t]{3}{*}{ ST239(24) } & III(18), NT(1) & t030(19) \\
\hline & $\mathrm{III}(4)$ & $\mathrm{t} 459(4)$ \\
\hline & III(1) & $\mathrm{t} 1510(1)$ \\
\hline \multirow[t]{3}{*}{ ST59(11) } & $\operatorname{IV}(8), \operatorname{II}(1)$ & $\mathrm{t} 437(9)$ \\
\hline & $\operatorname{IV}(1)$ & $\mathrm{t} 172(1)$ \\
\hline & $\operatorname{IV}(1)$ & $\mathrm{t} 441(1)$ \\
\hline \multirow[t]{3}{*}{ ST398(3) } & NT(1) & t034(1) \\
\hline & $\mathrm{V}(1)$ & $\mathrm{t} 5435(1)$ \\
\hline & $\mathrm{V}(1)$ & $\mathrm{t} 5462(1)$ \\
\hline \multirow[t]{3}{*}{ ST764(3) } & $\mathrm{IV}(1)$ & t002(1) \\
\hline & $\mathrm{II}(1)$ & t601(1) \\
\hline & $\mathrm{II}(1)$ & $\mathrm{t} 1084(1)$ \\
\hline \multirow[t]{2}{*}{ ST45(2) } & $\operatorname{IV}(1)$ & t116(1) \\
\hline & $\mathrm{IV}(1)$ & $\mathrm{t} 1510(1)$ \\
\hline ST6(1) & NT(1) & $\mathrm{t} 701(1)$ \\
\hline ST22(1) & $\mathrm{V}(1)$ & $\mathrm{t} 309(1)$ \\
\hline ST72(1) & IV(1) & t664(1) \\
\hline ST88(1) & II $(1)$ & $\mathrm{t} 3622(1)$ \\
\hline ST121(1) & $\mathrm{I}(1)$ & t2091(1) \\
\hline ST338(1) & $\mathrm{V}(1)$ & $\mathrm{t} 437(1)$ \\
\hline ST630(1) & $\mathrm{V}(1)$ & $\mathrm{t} 4549(1)$ \\
\hline ST845(1) & $\mathrm{III}(1)$ & $\mathrm{t} 084(1)$ \\
\hline ST965(1) & NT(1) & t062(1) \\
\hline
\end{tabular}

$M L S T$, multilocus sequence typing; spa, staphylococcal protein A; SCCmec, staphylococcus chromosomal cassette mec; $n$, number of isolates in each type; $N T$, non-typeable

nine remaining antibiotics, namely erythromycin (82.4\%), clindamycin (81.3\%), ciprofloxacin $(75.8 \%)$, levofloxacin $(74.7 \%)$, moxifloxacin $(73.6 \%)$, tetracycline $(67.0 \%)$, and gentamicin (56.0\%).

Compared with nSMRSA, SMRSA was more resistant to erythromycin, clindamycin, levofloxacin, tetracycline, moxifloxacin, and ciprofloxacin, but more susceptible to rifampicin. In addition, the SMRSA isolates had higher rates of multidrug resistance than the nSMRSA isolates. Among the nSMRSA isolates, we focused on ST239 and ST59, the major lineages found in Chinese settings. Similar to other nSMRSA strains, ST239 and ST59 were more susceptible to erythromycin, clindamycin, levofloxacin, tetracycline, moxifloxacin, and ciprofloxacin than SMRSA, but more resistant to rifampicin. However, no important difference was found in the multidrug resistance rate between SMRSA and ST239 or ST59 MRSA. 
Table 2 Antimicrobial resistance profiles of ST5SCCmecII- t2460MRSA and non-ST5-SCCmecII-t2460 MRSA

\begin{tabular}{lllllrr}
\hline Antibiotics $^{\mathrm{a}}$ & $\begin{array}{l}\text { SMRSA }^{\mathrm{b}} \\
(n=34) ; \mathrm{n}(\%)\end{array}$ & $\begin{array}{l}\mathrm{nSMRSA}^{\mathrm{b}} \\
(n=57) ; \mathrm{n}(\%)\end{array}$ & $\begin{array}{l}\text { ST239 and ST59 } \\
(n=35) ; \mathrm{n}(\%)\end{array}$ & $\begin{array}{l}\text { Total } \\
(n=91) ; \mathrm{n}(\%)\end{array}$ & $p^{\mathbf{c}}$ & $p^{\mathbf{d}}$ \\
\hline ERY & $34(100.0)$ & $41(71.9)$ & $25(71.4)$ & $75(82.4)$ & 0.001 & 0.002 \\
CLI & $34(100.0)$ & $40(70.2)$ & $25(71.4)$ & $74(81.3)$ & $<0.001$ & 0.002 \\
LEV & $34(100.0)$ & $34(59.6)$ & $25(71.4)$ & $68(74.7)$ & $<0.001$ & 0.002 \\
TET & $33(97.1)$ & $28(49.1)$ & $20(57.1)$ & $61(67.0)$ & $<0.001$ & $<0.001$ \\
RIF & $1(2.9)$ & $27(47.4)$ & $24(68.6)$ & $28(30.8)$ & $<0.001$ & $<0.001$ \\
GEN & $20(58.8)$ & $31(54.4)$ & $24(68.6)$ & $51(56.0)$ & 0.680 & 0.400 \\
MFX & $33(97.1)$ & $34(59.6)$ & $25(71.4)$ & $67(73.6)$ & $<0.001$ & 0.004 \\
CPFX & $34(100.0)$ & $35(61.4)$ & $26(74.3)$ & $69(75.8)$ & $<0.001$ & 0.005 \\
SXT & $0(0.0)$ & $1(1.8)$ & $0(0.0)$ & $1(1.1)$ & 1.000 & 1.000 \\
MDR & $34(100.0)$ & $47(82.5)$ & $31(88.6)$ & $81(89.0)$ & 0.025 & 0.130 \\
\hline
\end{tabular}

${ }^{a} E R Y$, erythromycin; $C L I$, clindamycin; $L E V$, levofloxacin; $T E T$, tetracycline; $R I F$, rifampicin; $G E N$, gentamicin; $M F X$, moxifloxacin; $C P F X$, ciprofloxacin; $S X T$, trimethoprim/sulfamethoxazole; $M D R$, multidrugresistant

${ }^{\mathrm{b}} \mathrm{n}$, number of isolates in each type

c $p, p$ value. The resistance rate to antibiotics in SMRSA isolates was compared with those in nSMRSA

${ }^{\mathrm{d}} p, p$ value. The resistance rate to antibiotics in SMRSA isolates was compared with those in ST239 and ST59

\section{Clinical characteristics}

The mean age of the patients was $61.87 \pm 15.39$ (mean $\pm \mathrm{SD}$ ) years and the age range was 28 to 97 years. Seventy $(76.9 \%)$ strains were from men and $21(23.1 \%)$ were from women. Of the MRSA isolates, 94.5\% (86/91) were HA-MRSA and 5.5\% (5/91) were CA-MRSA. The most common underlying diseases were cerebrovascular disease $(n=41 ; 45.1 \%)$, diabetes mellitus $(n=18 ; 19.8 \%)$, chronic renal disease $(n=17 ; 18.7 \%)$, malignancy $(n=16$; $17.6 \%)$, cardiovascular disease $(n=14 ; 15.4 \%)$, chronic pulmonary disease $(n=12 ; 13.2 \%)$, and hepatic disease $(n=11 ; 12.1 \%)$. As for the patients' outcomes, 62 patients (68.1\%) survived while the remaining 29 (31.9\%) died.

As described, we also collected blood indicators, PCT levels, and information on any invasive procedures and ICU hospitalization undergone by the patients to further compare the effects of SMRSA and nSMRSA infections. The clinical information is shown in Table 3. The sex, age, underlying diseases, and sources of infection were not very different between patients with SMRSA infections and those with nSMRSA infections. However, the PCT level, WBC count, frequency of ICU hospitalization, frequency of invasive procedures within 1 month, and 30-day mortality were higher in patients with SMRSA infections than in those with nSMRSA infections. According to a comparison of the clinical features and patient outcomes between patients infected with SMRSA and those infected with the specific nSMRSA lineages that are most common in Chinese settings (ST239 and ST59), patients with SMRSA infections had higher levels of WBCs, higher 30-day mortality, more frequent ICU hospitalization, and more frequent invasive procedures than those with ST239 and ST59 infections.

\section{Discussion}

S. aureus, especially MRSA, has been considered a serious threat to public health for several decades [1]. The predominant MLST of MRSA in China were previously reported to be ST239 [20-23] and ST59 [12]. However, according to the results of this study, in this hospital, SMRSA was the dominant MRSA, followed by ST239 and ST59. This finding is consistent with recent epidemiological changes in MRSA in China [9, 20]. SMRSA has rapidly increased in the recent past and become the predominant clone in some parts of China $[9,20]$, such as Wuhan where this study was carried out. Given the rapid increase in SMRSA infection rates among inpatients, it is urgently necessary to understand the prevalence, antimicrobial susceptibility, and clinical prognosis of SMRSA infections.

As a newly emerging clone, SMRSA prevalence has increased rapidly, but so far, SMRSA has been reported only in Asia, including China, South Korea, and Malaysia $[9,10,24-26]$. This may be due to the fact that many studies do not perform spa typing and/or because the frequency of SMRSA is limited in other regions. In South Korea, Kim et al. have identified SCVs in two patients who presented with MRSA bacteremia, nine blood isolates were collected and all isolates belonged to the same genotype (SMRSA) [27]. Our results showed that SMRSA clone is usually an SCV. SCVs in S. aureus are associated with intracellular persistence and reduced antimicrobial 
Table 3 Analysis of risk factors for ST5-t2460-SCCmecII MRSA infection

\begin{tabular}{|c|c|c|c|c|c|}
\hline & \multicolumn{4}{|c|}{$\mathrm{n}(\%) /$ mean $\pm \mathrm{SD} /$ median $(\mathrm{Q} 1-\mathrm{Q} 3)$} & \multirow[t]{2}{*}{$p^{\mathbf{a}}$} \\
\hline & SMRSA $(n=34)$ & nSMRSA $(n=57)$ & $\begin{array}{l}\text { ST239 and ST59 } \\
(n=35)\end{array}$ & Total $(n=91)$ & \\
\hline Sex, M/F & $28 / 6$ & $42 / 15$ & $25 / 10$ & $21 / 70$ & 0.3420 .282 \\
\hline Age, years & $63.97 \pm 16.48$ & $60.61 \pm 14.71$ & $61.40 \pm 14.73$ & $61.87 \pm 15.39$ & 0.3170 .497 \\
\hline PCT $(\mathrm{ng} / \mathrm{mL})^{*}$ & $0.66(0.19-2.54)$ & $0.21(0.06-1.61)$ & $0.41(0.06-1.71)$ & $0.41(0.12-1.71)$ & 0.0270 .146 \\
\hline \multicolumn{6}{|l|}{ Blood indicators* } \\
\hline $\mathrm{WBC}\left(\times 10^{9} / \mathrm{L}\right)$ & $12.54 \pm 6.61$ & $9.49 \pm 4.11$ & $9.62 \pm 3.96$ & $10.63 \pm 5.36$ & 0.0190 .031 \\
\hline $\mathrm{RBC}\left(\times 10^{12} / \mathrm{L}\right)$ & $3.06 \pm 0.71$ & $3.25 \pm 0.75$ & $3.27 \pm 0.83$ & $3.18 \pm 0.74$ & 0.2260 .247 \\
\hline $\operatorname{HGB}(\mathrm{g} / \mathrm{L})$ & $91.53 \pm 21.68$ & $96.68 \pm 20.84$ & $95.67 \pm 21.38$ & $94.76 \pm 21.18$ & 0.2630 .427 \\
\hline $\operatorname{PLT}\left(\times 10^{9} / \mathrm{L}\right)$ & $181.00(101.25-246.50)$ & $196(131.50-282.00)$ & $194.00(140.00-258.00)$ & $194.00(123.00-278.00)$ & 0.2970 .337 \\
\hline NEUT $(\%)$ & $85.30(78.58-89.25)$ & $81.50(74.95-89.40)$ & $83.60(75.00-90.00)$ & $82.80(75.10-89.30)$ & 0.2880 .862 \\
\hline LYMPH (\%) & $6.25(4.58-10.30)$ & $8.30(5.15-17.00)$ & $7.90(5.00-14.10)$ & $7.00(5.00-12.80)$ & 0.1110 .337 \\
\hline MONO (\%) & $7.83(5.98-11.71)$ & $7.40(4.90-9.95)$ & $6.60(5.00-9.00)$ & $7.50(5.30-10.10)$ & 0.4580 .208 \\
\hline $\begin{array}{l}\text { Healthcare-associated, } \\
\text { HA/CA }\end{array}$ & $34 / 0$ & $52 / 5$ & $32 / 3$ & $86 / 5$ & 0.1930 .248 \\
\hline \multicolumn{6}{|l|}{ Underlying disease } \\
\hline Cerebrovascular disease & $17(50.0)$ & $24(42.1)$ & $18(51.4)$ & $41(45.1)$ & 0.4640 .906 \\
\hline Diabetes mellitus & $7(20.6)$ & $11(19.3)$ & $10(28.6)$ & $18(19.8)$ & 0.8810 .442 \\
\hline Chronic renal disease & $7(20.6)$ & $10(17.5)$ & $7(20.0)$ & $17(18.7)$ & 0.7190 .952 \\
\hline Malignancy & $7(20.6)$ & $9(15.8)$ & $3(8.6)$ & $16(17.6)$ & 0.5610 .282 \\
\hline Cardiovascular disease & $7(20.6)$ & $7(12.3)$ & $5(14.3)$ & $14(15.4)$ & 0.2880 .490 \\
\hline $\begin{array}{l}\text { Chronic pulmonary } \\
\text { disease }\end{array}$ & 7 (20.6) & $5(8.8)$ & $7(20.0)$ & $12(13.2)$ & 0.1970 .952 \\
\hline Hepatic disease & $6(17.6)$ & $5(8.8)$ & $4(11.4)$ & $11(12.1)$ & 0.3550 .695 \\
\hline \multicolumn{6}{|l|}{ Source of infection } \\
\hline Skin/soft tissue & $1(2.9)$ & $9(15.8)$ & $5(14.3)$ & $10(11.0)$ & 0.1210 .213 \\
\hline Sputum & $16(47.1)$ & $22(38.6)$ & $13(37.1)$ & $38(41.8)$ & 0.4280 .404 \\
\hline Blood & $11(32.4)$ & $9(15.8)$ & $7(20.0)$ & $20(22.0)$ & 0.0650 .243 \\
\hline Other & $6(17.6)$ & $17(29.8)$ & $10(28.6)$ & $23(25.3)$ & 0.1960 .282 \\
\hline ICU hospitalization & $28(82.4)$ & $23(40.4)$ & $16(45.7)$ & $51(56.0)$ & $<0.0010 .002$ \\
\hline Invasive procedure & $33(97.1)$ & $37(64.9)$ & $24(68.6)$ & $70(76.9)$ & $<0.0010 .002$ \\
\hline 30-day mortality & $18(52.9)$ & $11(19.3)$ & $6(17.1)$ & $29(31.9)$ & $<0.0010 .025$ \\
\hline
\end{tabular}

$M$, male; $F$, female; $S D$, standard deviation; $P C T$, procalcitonin; $W B C$, white blood cell; $R B C$, red blood cell; $H G B$, hemoglobin; $P L T$, platelets; $N E U T$, neutrophil; $L Y M P H$, lymphocyte; $M O N O$, monocytes; $H A$, hospital-acquired; $C A$, community-acquired; $I C U$, intensive care unit

*The reference values of PCT and blood indicators: PCT $<0.05 \mathrm{ng} / \mathrm{mL} ;$ WBC $(3.5-9.5) \times 10^{9} / \mathrm{L} ; \mathrm{RBC}(4.3-5.8) \times 10^{12} / \mathrm{L}(\mathrm{male}),(3.8-$ $5.1) \times 10^{12} / \mathrm{L}$ (female); HGB (130-175) g/L (male), (115-150) g/L (female); PLT $(125-350) \times 10^{9} / \mathrm{L} ;$ NEUT (40-75)\%; LYMPH (20-50)\%; MONO (3-10)\%

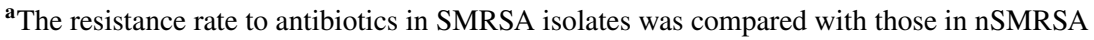

${ }^{\mathbf{b}}$ The resistance rate to antibiotics in SMRSA isolates was compared with those in ST239 and ST59

susceptibility, which can lead to persistent and recurrent $S$. aureus infection $[27,28]$. Consistent with the report from South Korea [27], all of the SMRSA strains in the present study had the characteristics of SCVs. The comparison of the antimicrobial resistance profiles of SMRSA and nSMRSA showed that the former had much wider resistance, which is consistent with a previous study [27, 29] and may provide a plausible explanation for the rapid increase of SMRSA strains in recent years. Multidrug resistance makes SMRSA strains more competitive in the hospital setting. Compared with ST239 and ST59, which were previously the predominant lineages, the multiple resistance of SMRSA to erythromycin, clindamycin, levofloxacin, tetracycline, moxifloxacin, and ciprofloxacin may be one of the reasons why SMRSA is gradually replacing ST239-MRSA. However, compared with ST239 and ST59 or with other nSMRSA strains, the resistance rate of SMRSA to rifampicin was lower. Considering that the use of rifampicin alone to treat MRSA infections can easily lead to resistance [30], it is suggested that 
rifampicin combined with other antibiotics should be considered when conventional antibiotics fail to treat SMRSA infections.

Although the incidence of MRSA infections has recently declined in some regions, these bacteria still pose a serious threat to public health, with high mortality rates. In our study, the 30-day mortality was $31.9 \%$ in MRSA-infected patients, which was much higher than that reported in other studies [31]. Specifically, the 30-day mortality of nSMRSA patients was similar to those observed in previous studies [31, 32], but that of SMRSA patients was $52.9 \%$, which is much higher than that from the main lineages in Chinese settings (ST239 and ST59) and other nSMRSA strains. These results may suggest that SMRSA strains are more virulent than nSMRSA and also explain why SMRSA is gradually replacing ST239 as the predominant MRSA strain. Taking into consideration the results of previous studies of the molecular characteristics of $S$. aureus and the outcome of infections [6, 7, 33], it is reasonable to conclude that the molecular characteristics of different $S$. aureus strains may affect the outcome of infections. Specifically, we found that SMRSA, an emerging clone in China, was associated with high 30-day mortality. Moreover, some device-related infections caused by $S$. aureus SCVs have previously been reported [34, 35]. This is consistent with our finding that SMRSA infections were associated with more frequent ICU hospitalizations and invasive procedures such as venous catheterization and tracheostomy, in which SCVs might have played a role in causing device-related infections. These results seem to suggest that SMRSA infection is related to worsening of patients' condition. In view of this, it is not difficult to understand why SMRSA strains are hospital-acquired, as described in various studies [8-10].

The PCT level and WBC count are inflammatory biomarkers that reflect the underlying biological processes as well as disease severity. For instance, the levels of PCT may rise dramatically in cases of bacterial infection, with higher values correlating with more severe infection [36, 37]. It was reported that PCT plays an important role in the inflammatory responses and histopathological changes in $S$. aureus infections. High levels of inflammatory biomarkers on admission, particularly PCT, are associated with a higher likelihood of S. aureus infection [38], and a higher risk of inhospital mortality among patients [39]. In our study, patients infected with MRSA infections had higher levels of PCT. Moreover, the PCT levels and WBC counts were higher in patients with SMRSA infections than in those with nSMRSA infections. Taking into consideration the high fatality rate together with multiple antibiotic resistance of SMRSA, we conclude that SMRSA may be linked with more serious infections and poorer prognosis than other strains and we should pay more attention to the spread of SMRSA.
Our study has some limitations. First, we ran univariate statistical analysis with small sample size, which may have caused information bias. Second, the retrospective collection of data and lack of some inflammatory biomarkers, such as interleukin 6, C-reactive protein, and erythrocyte sedimentation rate, limited the informativeness. Third, the study was performed in a single center, which prevents broad representativeness. Thus, appropriate caution should be taken when interpreting our data.

\section{Conclusion}

In this study, SMRSA was the predominant clone, followed by ST239-SCCmecIII-t030 and ST59-SCCmecIV-t437. Phenotypically, SMRSA was an SCV of S. aureus and was associated with multiple antibiotic resistance, but was susceptible to rifampicin. SMRSA strains were also related to serious infections and poor prognosis. Patients with SMRSA had higher 30-day mortality, increased levels of inflammatory biomarkers, and more frequent ICU hospitalization and invasive procedures.

Acknowledgements We are grateful to Zhongnan Hospital of Wuhan University for providing clinical isolates and data.

Author contribution YRL and RF designed the studies and obtained funding; XHL, JZ, and JYZ performed the experiments; YFZ and XWL performed the statistical analysis; XHL wrote the manuscript; JZ contributed to manuscript revision. All authors read and approved the submitted version.

Funding This work was supported by the National Science and Technology Major Projects of China (no. 2017ZX10103005 and no. 2020ZX09201007). The funding bodies had no role in the design of the study and collection, analysis, and interpretation of data and in writing the manuscript.

Data availability The data of this study are presented in the article text and tables. Additional details are available by contacting the corresponding author upon reasonable request.

\section{Declarations}

Ethics approval. This study was approved by the Ethics Committee of Zhongnan Hospital, which waived the requirement for informed consent because the details of patients with $S$. aureus infection were anonymized.

Conflict of interest. The authors declare no competing interests.

Open Access This article is licensed under a Creative Commons Attribution 4.0 International License, which permits use, sharing, adaptation, distribution and reproduction in any medium or format, as long as you give appropriate credit to the original author(s) and the source, provide a link to the Creative Commons licence, and indicate if changes were made. The images or other third party material in this article are 
included in the article's Creative Commons licence, unless indicated otherwise in a credit line to the material. If material is not included in the article's Creative Commons licence and your intended use is not permitted by statutory regulation or exceeds the permitted use, you will need to obtain permission directly from the copyright holder. To view a copy of this licence, visit http://creativecommons.org/licenses/by/4.0/.

\section{References}

1. Sikorska H, Smoragiewicz W (2013) Role of probiotics in the prevention and treatment of meticillin-resistant Staphylococcus aureus infections. Int J Antimicrob Agents 42(6):475-481. https:// doi.org/10.1016/j.ijantimicag.2013.08.003

2. Su CH, Chang SC, Yan JJ, Tseng SH, Chien LJ, Fang CT (2013) Excess mortality and long-term disability from healthcare-associated Staphylococcus aureus infections: a population-based matched cohort study. PLoS One 8(8):e71055. https://doi.org/10. 1371/journal.pone.0071055

3. Deresinski S (2005) Methicillin-resistant Staphylococcus aureus: an evolutionary, epidemiologic, and therapeutic odyssey. Clin Infect Dis 40(4):562-573. https://doi.org/10.1086/427701

4. Peacock SJ, Paterson GK (2015) Mechanisms of methicillin resistance in Staphylococcus aureus. Annu Rev Biochem 84:577-601. https://doi.org/10.1146/annurev-biochem-060614-034516

5. Xiao M, Wang H, Zhao Y, Mao LL, Brown M, Yu YS, O'Sullivan MV, Kong F, Xu YC (2013) National surveillance of methicillinresistant Staphylococcus aureus in China highlights a still-evolving epidemiology with 15 novel emerging multilocus sequence types. J Clin Microbiol 51(11):3638-3644. https://doi.org/10. 1128/JCM.01375-13

6. Rao Q, Shang W, Hu X, Rao X (2015) Staphylococcus aureus ST121: a globally disseminated hypervirulent clone. J Med Microbiol 64(12):1462-1473. https://doi.org/10.1099/jmm.0.000185

7. Bouiller K, Gbaguidi-Haore H, Hocquet D, Cholley P, Bertrand X, Chirouze C (2016) Clonal complex 398 methicillin-susceptible Staphylococcus aureus bloodstream infections are associated with high mortality. Clin Microbiol Infect 22(5):451-455. https://doi. org/10.1016/j.cmi.2016.01.018

8. Li Y, Zhao R, Zhang X, Han Q, Qian X, Gu G, Shi J, Xu J (2015) Prevalence of enterotoxin genes and spa genotypes of methicillinresistant Staphylococcus aureus from a tertiary care hospital in China. J Clin Diagn Res 9(5):DC11-14. https://doi.org/10.7860/ JCDR/2015/12090.5990

9. Dai Y, Liu J, Guo W, Meng H, Huang Q, He L, Gao Q, Lv H, Liu Y, Wang Y, Wang H, Liu Q, Li M (2019) Decreasing methicillinresistant Staphylococcus aureus (MRSA) infections is attributable to the disappearance of predominant MRSA ST239 clones, Shanghai, 2008-2017. Emerg Microbes Infect 8(1):471-478. https://doi. org/10.1080/22221751.2019.1595161

10. Li X, Fang F, Zhao J, Lou N, Li C, Huang T, Li Y (2018) Molecular characteristics and virulence gene profiles of Staphylococcus aureus causing bloodstream infection. Braz J Infect Dis 22(6):487-494. https://doi.org/10.1016/j.bjid.2018.12.001

11. Kim T, Yi J, Hong KH, Park JS, Kim EC (2011) Distribution of virulence genes in spa types of methicillin-resistant Staphylococcus aureus isolated from patients in intensive care units. Korean $\mathbf{J}$ Lab Med 31(1):30-36. https://doi.org/10.3343/kjlm.2011.31.1.30

12. Wang X, Li X, Liu W, Huang W, Fu Q, Li M (2016) Molecular characteristic and virulence gene profiles of community-associated methicillin-resistant Staphylococcus aureus isolates from pediatric patients in Shanghai. China Front Microbiol 7:1818. https://doi.org/10.3389/fmicb.2016.01818
13. Li T, Lu H, Wang X, Gao Q, Dai Y, Shang J, Li M (2017) Molecular characteristics of Staphylococcus aureus causing bovine mastitis between 2014 and 2015. Front Cell Infect Microbiol 7:127. https://doi.org/10.3389/fcimb.2017.00127

14. Luo K, Shao F, Kamara KN, Chen S, Zhang R, Duan G, Yang H (2018) Molecular characteristics of antimicrobial resistance and virulence determinants of Staphylococcus aureus isolates derived from clinical infection and food. J Clin Lab Anal 32(7):e22456. https://doi.org/10.1002/jcla.22456

15. Zhang K, McClure JA, Elsayed S, Louie T, Conly JM (2005) Novel multiplex PCR assay for characterization and concomitant subtyping of staphylococcal cassette chromosome mec types I to $\mathrm{V}$ in methicillin-resistant Staphylococcus aureus. J Clin Microbiol 43(10):5026-5033. https://doi.org/10.1128/JCM.43.10.50265033.2005

16. Li X, Huang T, Xu K, Li C, Li Y (2019) Molecular characteristics and virulence gene profiles of Staphylococcus aureus isolates in Hainan. China BMC Infect Dis 19(1):873. https://doi. org/10.1186/s12879-019-4547-5

17. Boye K, Bartels MD, Andersen IS, Moller JA, Westh H (2007) A new multiplex PCR for easy screening of methicillin-resistant Staphylococcus aureus SCCmec types I-V. Clin Microbiol Infect 13(7):725-727. https://doi.org/10.1111/j.1469-0691.2007. 01720.x

18. CLSI e (2019) Performance standards for antimicrobial suscepetibility testing, 29th ed. CLSI supplement M100. Wayne: Clinical and Laboratory Standards Institute

19. Naimi TS, LeDell KH, Como-Sabetti K, Borchardt SM, Boxrud DJ, Etienne J, Johnson SK, Vandenesch F, Fridkin S, O’Boyle C, Danila RN, Lynfield R (2003) Comparison of community- and health care-associated methicillin-resistant Staphylococcus aureus infection. JAMA 290(22):2976-2984. https://doi.org/10.1001/ jama.290.22.2976

20. Chen H, Liu Y, Jiang X, Chen M, Wang H (2010) Rapid change of methicillin-resistant Staphylococcus aureus clones in a Chinese tertiary care hospital over a 15 -year period. Antimicrob Agents Chemother 54(5):1842-1847. https://doi.org/10.1128/AAC. 01563-09

21. Cheng H, Yuan W, Zeng F, Hu Q, Shang W, Tang D, Xue W, Fu J, Liu J, Liu N, Zhu J, Yang J, Hu Z, Yuan J, Zhang X, Li S, Chen Z, Hu X, Rao X (2013) Molecular and phenotypic evidence for the spread of three major methicillin-resistant Staphylococcus aureus clones associated with two characteristic antimicrobial resistance profiles in China. J Antimicrob Chemother 68(11):2453-2457. https://doi.org/10.1093/jac/dkt213

22. Shang W, Hu Q, Yuan W, Cheng H, Yang J, Hu Z, Yuan J, Zhang X, Peng H, Yang Y, Hu X, Li M, Zhu J, Rao X (2016) Comparative fitness and determinants for the characteristic drug resistance of ST239-MRSA-III-t030 and ST239-MRSA-III-t037 strains isolated in China. Microb Drug Resist 22(3):185-192. https://doi.org/ 10.1089/mdr.2015.0226

23. Yu Y, Yao Y, Weng Q, Li J, Huang J, Liao Y, Zhu F, Zhao Q, Shen X, Niu J (2017) Dissemination and molecular characterization of Staphylococcus aureus at a tertiary referral hospital in Xiamen city. China Biomed Res Int 2017:1367179. https://doi.org/10. 1155/2017/1367179

24. Yu F, Liu Y, Lu C, Lv J, Qi X, Ding Y, Li D, Huang X, Hu L, Wang L (2015) Dissemination of fusidic acid resistance among Staphylococcus aureus clinical isolates. BMC Microbiol 15:210. https://doi.org/10.1186/s12866-015-0552-Z

25. Ho WY, Choo QC, Chew CH (2017) Predominance of three closely related methicillin-resistant Staphylococcus aureus clones carrying a unique $c c r C$-positive SCCmec type III and the emergence of spa 304 and t690 SCCmec type IV pvl(+) MRSA isolates in Kinta Valley. Malaysia Microb Drug Resist 23(2):215223. https://doi.org/10.1089/mdr.2015.0250 
26. Mun YS, Hwang YJ (2019) Novel spa and Multi-Locus Sequence Types (MLST) of Staphylococcus aureus samples isolated from clinical specimens in Korean. Antibiotics (Basel) 8 (4). https:// doi.org/10.3390/antibiotics8040202

27. Kim NH, Kang YM, Han WD, Park KU, Park KH, Yoo JI, Lee DG, Park C, Song KH, Kim ES, Park SW, Kim NJ, Oh MD, Kim HB (2016) Small-colony variants in persistent and recurrent Staphylococcus aureus bacteremia. Microb Drug Resist 22(7):538-544. https://doi.org/10.1089/mdr.2015.0262

28. Kahl BC (2014) Small colony variants (SCVs) of Staphylococcus aureus - a bacterial survival strategy. Infect Genet Evol 21:515522. https://doi.org/10.1016/j.meegid.2013.05.016

29. von Eiff C, Proctor RA, Peters G (2000) Small colony variants of Staphylococci: a link to persistent infections. Berl Munch Tierarztl Wochenschr 113(9):321-325

30. Zhao X, Drlica K (2001) Restricting the selection of antibioticresistant mutants: a general strategy derived from fluoroquinolone studies. Clin Infect Dis 33(Suppl 3):S147-156. https://doi.org/10. 1086/321841

31. Ayau P, Bardossy AC, Sanchez G, Ortiz R, Moreno D, Hartman P, Rizvi K, Prentiss TC, Perri MB, Mahan M, Huang V, Reyes K, Zervos MJ (2017) Risk factors for 30-day mortality in patients with methicillin-resistant Staphylococcus aureus bloodstream infections. Int J Infect Dis 61:3-6. https://doi.org/10.1016/j.ijid. 2017.05.010

32. Simor AE, Pelude L, Golding G, Fernandes R, Bryce E, Frenette C, Gravel D, Katz K, McGeer A, Mulvey MR, Smith S, Weiss K, Program CNIS (2016) Determinants of outcome in hospitalized patients with methicillin-resistant Staphylococcus aureus bloodstream infection: results from national surveillance in Canada, 2008-2012. Infect Control Hosp Epidemiol 37(4):390-397. https://doi.org/10.1017/ice.2015.323

33. Fowler VG Jr, Nelson CL, McIntyre LM, Kreiswirth BN, Monk A, Archer GL, Federspiel J, Naidich S, Remortel B, Rude T, Brown P, Reller LB, Corey GR, Gill SR (2007) Potential associations between hematogenous complications and bacterial genotype in
Staphylococcus aureus infection. J Infect Dis 196(5):738-747. https://doi.org/10.1086/520088

34. Seifert H, Wisplinghoff H, Schnabel P, von Eiff C (2003) Small colony variants of Staphylococcus aureus and pacemaker-related infection. Emerg Infect Dis 9(10):1316-1318. https://doi.org/10. 3201/eid0910.0302000

35. Spanu T, Romano L, D’Inzeo T, Masucci L, Albanese A, Papacci F, Marchese E, Sanguinetti M, Fadda G (2005) Recurrent ventriculoperitoneal shunt infection caused by small-colony variants of Staphylococcus aureus. Clin Infect Dis 41(5):e48-52. https:// doi.org/10.1086/432577

36. Cuculi F, Toggweiler S, Auer M, der Maur ChA, Zuber M, Erne $\mathrm{P}$ (2008) Serum procalcitonin has the potential to identify Staphylococcus aureus endocarditis. Eur J Clin Microbiol Infect Dis 27(11):1145-1149. https://doi.org/10.1007/s10096-008-0541-3

37. Cornelissen CG, Frechen DA, Schreiner K, Marx N, Kruger S (2013) Inflammatory parameters and prediction of prognosis in infective endocarditis. BMC Infect Dis 13:272. https://doi.org/10. 1186/1471-2334-13-272

38. Wu D, Zhou S, Hu S, Liu B (2017) Inflammatory responses and histopathological changes in a mouse model of Staphylococcus aureus-induced bloodstream infections. J Infect Dev Ctries 11(4):294-305. https://doi.org/10.3855/jidc.7800

39. Tascini C, Aimo A, Arzilli C, Sbrana F, Ripoli A, Ghiadoni L, Bertone C, Passino C, Attanasio V, Sozio E, Taddei E, Murri R, Fantoni M, Paciosi F, Francisci D, Pasticci MB, Pallotto C, Di Caprio G, Carozza A, Maffei S, Emdin M (2020) Procalcitonin, white blood cell count and C-reactive protein as predictors of S. aureus infection and mortality in infective endocarditis. Int $\mathrm{J}$ Cardiol 301:190-194. https://doi.org/10.1016/j.ijcard.2019.08.013

Publisher's note Springer Nature remains neutral with regard to jurisdictional claims in published maps and institutional affiliations. 